\title{
Referral Finder: Saving Time and Improving The Quality of In-hospital Referrals
}

\author{
Jennifer Cathcart, Neil Cowan, Vicki Tully
}

Ninewells Hospital, Dundee, Scotland

\begin{abstract}
Making referrals to other hospital specialties is one of the key duties of the foundation doctor, which can be difficult and time consuming. In Ninewells hospital, Scotland, in our experience the effectiveness of referrals is limited by contact details not being readily accessible and foundation doctors not knowing what information is relevant to each specialty.
\end{abstract}

We surveyed foundation doctors on their experience of the existing referral process to identify where we needed to focus to improve the process. The doctors reported significant delays in obtaining contact details from the operator, and found they did not know the specific information needed in each referral.

To increase the information available to foundation doctors, we set up a page on the staff intranet called 'Referral Finder'. This page includes contact details, guidelines for referral, and links to relevant protocols for each specialty. By making this information readily accessible our objective was to increase the speed and quality of referrals.

When surveyed two months after the web page was established, foundation doctors reported a reduction in calls to operator from baseline and reported achieving more effective referrals. When asked to comment, many doctors asked if the page could include details for other hospitals in our health board and provide more specialty specific information.

This feedback prompted us to extend the scope of the page to include the district general hospital in our region, and update many of the existing details. Doctors were then surveyed after the updates, $100 \%$ agreed that the website saved time and there was a $49.3 \%$ reduction in doctors who reported not knowing the specific information needed for a referral.

Having adequate information improved referrals and resulted in time saved. This would allow more time for patient care. The quality improvement project was praised among doctors as a useful, innovative and replicable project.

\section{Problem}

In Ninewells hospital, Dundee, Scotland, foundation doctors spent considerable time accessing referral contact details via the operator. This resulted in significant delays which impacted on other ward jobs and patient care. After obtaining the contact details from the operator, more time was spent accumulating patient information by the doctor to make the referral. Despite this, referrals were often lacking specific information resulting in inadequate referrals. Ninewells is a large teaching hospital with 26 specialties where the referral process, and the specific information required for referral, varies between each specialty. Existing paper records of referral contact details in the wards were incomplete and were not kept updated. In addition, there was no guidance on what to include in the referral for any specialty. As two foundation doctors, we had encountered frustration at wasted time, incomplete referrals, and patient safety issues caused by the lack of available referral information. We felt a simple online page where referral information was kept would mean the information could be accessed universally throughout the hospital. The aim of the project was to improve the speed and quality of referrals.

\section{Background}

Making referrals to other specialties is a regular part of the foundation doctor workload.[1] The foundation programme curriculum, which is set by the General Medical Council (GMC)[2] lists making an appropriate referral within the hospital as a core competency. One audit in the emergency department identified that the majority of its doctors, of whom most were equivalent grades of doctor to foundation year two doctors, found referrals to be problematic. Two of the reasons given was their own lack of knowledge of the subject and lack of protocols for referral.[3] Referrals form part of a foundation doctor's potentially extensive workload,[4-6] which can lead to stress and fatigue[6] and then to sub-optimal patient care.[7]

The aim of our project was to tackle both these issues; reduce the time to complete referrals and to improve the quality of referrals between hospital specialties, in order to improve patient care. The latter point is reinforced by several studies which show that poor communication can compromise patient safety.[8-11] One study looking at referrals from the emergency department to the medical floor found that variations in the clinical information required 
between the specialties was one of the main factors that attributed to the difficulty in negotiating patient transfers.[12] It was also noted within this study that the timeliness of the referral also impacted on its success.

There are limited reported projects trying to solve this problem. One similar project is reported in the British Medical Journal (BMJ) Quality Improvement journal in which a website was used to disseminate contact information regarding referrals.[13] A positive outcome resulted, and junior doctors contacted their senior less for information on how to refer as a result. However, the project gave only contact methods to refer while our project extends beyond access to correct contact details by giving guidelines for specific supporting information needed in a referral. Furthermore, they used a password protected external site whereas our approach used the National Health Service (NHS) intranet to host the site. Password protection introduces more clicks and increases time to access, which can affect access as shown by a project in Bristol, which showed that a simple re-organisation of the intranet sites to reduce the number of clicks meant more doctors could find the correct guidelines.[14]

\section{Baseline measurement}

Before implementation of the intranet page, we collected baseline data via a questionnaire put to foundation doctors. We distributed this at a foundation teaching session in electronic form, via email and via the foundation doctor social media page. We asked all foundation doctors, even those not based in Ninewells at present, as all doctors rotate though Ninewells hospital. We collected four key baseline measurements for this project which were used in the subsequent questionnaires to directly compare the results to baseline. These were:

1. Number of calls to the operator

2. Time gathering information for a referral

3. Number of doctors who report times not having information requested to hand when referring

4. Number of doctors who report not knowing the specific information required by each specialty

The questionnaire had multiple choice answers. In addition to the above measures, doctors were asked how many times and for what reason they phone the operator. We also gauged issues with patient care by asking the group how far they agreed that there were times where their patient's treatment has been delayed whilst awaiting referral details from the operator.

75 foundation doctors replied to the questionnaire out of 187 . This found that $94 \%$ of foundation doctors used the operator to get contact details for a referral to a specialty and $79 \%$ called the operator three times or more per day. $47 \%$ of doctors surveyed reported that calls took 2-3 minutes to be answered when they called the operator with $25.8 \%$ reporting that calls took more than five minutes to be answered. $73.9 \%$ of doctors felt that the time taken on the phone to the operator at times leads to patients' treatment being delayed. $73.8 \%$ of doctors spent more than five minutes gathering information to make a referral. $83.1 \%$ of doctors agreed that there were times they did not have information requested by the specialist to hand. $57.8 \%$ felt they did not know the specific details each specialty requires for referral. $97 \%$ of doctors considered the creation of a referral finder page to be a helpful idea.

\section{Design}

We designed a page to be placed on our hospital intranet; staffnet. We felt an intranet page would easy to use, easy to update, and cost-effective. This provided several advantages over paper lists which are easily lost and cost more to reproduce. We sought informal verbal advice from colleges on the design of the intranet page which includes the following for each specialty;

Preferred contact method

Contact details

Best time to refer

Details required for effective referral

Guidelines to be aware of

Other helpful information

We sent out emails to registrars who worked on call in each specialty to fill out each of the sections above. The registrars included links to other guidelines or protocols on the intranet which could help foundation doctors without the need to phone the specialist. For example, renal protection guidelines, our hospital antibiotic guidelines and gastroenterology procedure guidelines.

The page was created as a HTML document with a list of specialties at the top, each of which linked to specific guidance when clicked on. The page was titled "Referral Finder". This document was then uploaded to a section of the intranet which is easily accessible to foundation doctors. It is reviewed and updated every four months by an annually elected foundation doctor group. Referral Finder will continue to be used in Ninewells hospital as long as it deemed useful as determined by an annual questionnaire whose results will be analysed by the foundation doctor group.

\section{Strategy}

PDSA Cycle 1: Baseline data showed that foundation doctors agreed that an intranet page with guidance on information to include in a referral and contact numbers would be helpful. The Referral Finder page was created and initially shown to couple of foundation doctors. They gave verbal feedback that the page was easy to use and felt the information supplied by each specialty was sufficient. After using it, they agreed that they were able to phone the operator less and make better referrals. No formal data was collected at this stage.

PDSA Cycle 2: The Referral Finder was then distributed among the 
foundation doctors within Ninewells hospital. It was launched at a teaching session and an email was sent to those not able to attend the teaching session. After two months of the page being available for use, a questionnaire was issued. The results showed a significant improvement from baseline. Doctors reported saving time and making better referrals. The foundation doctors asked for the page to include more specialties such as nurse-led specialties.

PDSA Cycle 3: The Referral Finder was updated with more referral specialties added to include specialist nurses. Spelling errors were corrected as requested by user feedback from the questionnaires, two of which were in emails used for referrals. Informal user feedback via email to ourselves was positive about the updates. Due to its popularity, Referral Finder, in addition to being on Staffnet was added to the new junior doctor handbook launched in August. It was then further updated as requested in the questionnaire to include a neighbouring hospital Perth Royal Infirmary. A further questionnaire was sent out after the updates. This was sent out via email, on Facebook, and on the Staffnet, as well as handed out at a foundation teaching event. Again the positive results proved Referral Finder to be a useful intervention that saved time. It especially showed significant improvement in doctors reporting knowledge of specific information needed to refer. In addition, the results showed that after the updates, doctors rated the site more useful. Feedback pointed out some missing contact information in the Perth section which we are working on at present.

\section{Results}

In May 2015, after two months of Referral Finder being live on the intranet, we distributed the PSDA cycle 2 questionnaire via email; 33 foundation doctors completed this questionnaire. A PDSA cycle 3 questionnaire was sent out via email, posted on a Dundee foundation trainee social media page and distributed at a foundation trainee teaching session eight months later. This was distributed after updating the website and 61 foundation trainees responded. Both PDSA cycle 2 and cycle 3 questionnaires were completed only by those who used the Referral Finder page. We were unable to measure how many individual foundation doctors were using the website therefore we cannot comment on the response rate of either questionnaire.

Overall the project showed an improvement from baseline and has shown improvement from one cycle to the next. In the PDSA cycle 2 questionnaire, $73.5 \%$ of doctors report using the intranet page at least every week with $23.5 \%$ of doctors reporting using it every day. In PDSA cycle 3 questionnaire, weekly use had increased to $91.8 \%$ and daily use had increased from $23.5 \%$ to $47.5 \%$. In the PDSA cycle 2 questionnaire $90.9 \%$ doctors rated the usefulness at least $7 / 10$ and in the PSDA cycle 3 questionnaire $100 \%$ rated Referral Finder at least $7 / 10$.

The intranet page use has reduced the number of calls to the operator. These calls were reported by foundation doctors in the baseline questionnaire to be time-costly and a delay to patients' treatment. $79 \%$ of foundation doctors initially made more than three calls a day compared to $50.8 \%$ in the PSDA cycle 3 questionnaire. $37.5 \%$ called the operator more than three times a day in the PSDA cycle 2 questionnaire but this number may be likely due to the lower uptake of the questionnaire and not reflective of the true population. Regardless, an improvement from baseline is seen. Referral Finder on the other hand is shown to be quick to access and to save time as reported by foundation doctors. In both questionnaires the majority of doctors report that referral finder takes less than a minute to access (51.5\% and then $61.7 \%$ respectively). In PDSA cycle 2 questionnaire, $88 \%$ of foundation doctors agreed that Referral Finder saved time and this increased to $100 \%$, in PSDA cycle 3 , after updating the website. The time to gather information for a referral stayed roughly the same however with $72.1 \%$ reporting to take more than five minutes in the PDSA cycle 3 questionnaire compared to $73.8 \%$ at baseline.

With regards to the quality of referrals, there was a reported improvement in the knowledge of information required by each specialty with the use of Referral Finder. $57.8 \%$ of doctors at baseline reported a lack of knowledge of specific information required by each specialty, this improved to only $32.2 \%$ in the PDSA cycle 2 questionnaire, and just $8.5 \%$ in the PSDA cycle 3 questionnaire. $83.1 \%$ of doctors at baseline reported there were times they didn't have information requested to hand when referring, however following the introduction of the website, this has reduced to $63.3 \%$ in the PDSA cycle 3 questionnaire.

Data was collected on how much the page was viewed per month, as shown in the attached graph. The website has had a steady increase in number of views from 374 in it's first month, March 2015 to 1,783 in February 2016. This illustrates its overall popularity and points towards how helpful the foundation doctors find the site. The attachment also contains a table of the four measurements taken across the three questionnaire for comparison.

See supplementary file: ds7495.docx - "ReferralFinderResults"

\section{Lessons and limitations}

One of the limitations of the project was the lack of uptake of all the questionnaires for numerous reasons. This could have some influence on the results of the project as it may not be a true reflection of the whole population of foundation doctors. We learnt from this project that foundation doctors do not always engage with projects via emails. We had more uptake of the questionnaire when we posted it to our foundation year social media page and when we collected the questionnaire at a teaching session in person. There were doctors who had not heard of the site, they would have not been able to complete the PDSA cycle 2 or 3 questionnaires. These doctors could have missed the earlier teaching session and not read their emails. More could have been done to raise the awareness of the project early on, perhaps by creating posters placed round the hospital. We were able to see the number of views of the page, however it would have been useful to measure how many of the foundation doctors were using the Referral Finder page so we could gauge the response rates to PDSA cycle 2 and 3 questionnaires. This would have allowed for better interpretation of the results.

In hindsight we should have done more formal small scale testing 
i.e. within a ward setting before distributing it across the hospital. This may have allowed us to identify some of the missing contact information and spelling errors before it was widely distributed. We were able to correct minor errors after comments from users such as errors in email addresses after the second questionnaire but this may have been avoidable.

We did not survey the registrars receiving the referral within this project though they also stood to gain from the project. The improvement of the quality of referrals may have reduced their time taking the referral and added to their ability to make decisions. In addition easier access to the guidelines by the foundation doctor could have reduced the number of calls needed to the specialist. Another measurement which would be beneficial to measure would be the impact of saving time on patient care. Saving time allows more time for patient care and faster availability of referral information allows for a quicker response and advice from the registrar to help with patient care. These could be looked at in similar future projects.

\section{Conclusion}

Our baseline questionnaire highlighted that there was a lack of knowledge in information needed in a referral by foundation doctors and the task was time consuming causing delays to patient care. Our results showed that having referral information available as an intranet page is quicker to access than the operator, and the foundation doctors universally reported that they saved time by using it. This time saved allows more time for other tasks reducing large workloads which can lead to sub-optimal patient care.[7] This project reflects the success of a similar project giving online contact information for referrals.[13] However by also adding in guidance this project sought to improve the quality of referrals. The quality was improved with less foundation doctors lacking the information requested when making a referral. More doctors reported an improvement in knowledge of specific information needed for the referral when using Referral Finder. Better communication though better referrals reduces medical error.[8-11] As one of the key duties of the Foundation Doctor, it is likely that those in other hospitals have similar problems with referrals, as noted in studies.[3,12] Referral finder is a highly replicable project with low running and setting up cost with benefit to both patients and doctors. It is easily sustainable, this project will be maintained by a group of annually appointed foundation trainees who will update the guidance every four months. It has proven to improve the speed and quality of referrals.

\section{References}

1 Vance G, Burford B, Jandial S, and Scott J. Identifying the work activities performed by doctors in the foundation programme. Sept 2015. URL:

http://www.gmc-uk.org/Activities_of_FP_doctors_full_report_FINAL 210915.pdf 62792484.pdf

2 GMC. The UK foundation programme curriculum. 2012. updated for August 2015. URL: http://www.foundationprogramme.nhs.uk/pages/home/curriculumand-assessment/curriculum2012

3 Reid C, Moorthy C, and Forshaw K. Referral patterns: an audit into referral practice among doctors in emergency medicine. Emerg Med J 2005;22:355-358

4 Nason G, Liddy S, Murphy T, and Doherty E. A cross-sectional observation of burnout in a sample of Irish junior doctors. Irish $\mathrm{J}$ Med Sci 2013; 182(4):595-9.

5 Gunasingam N, Burns K, Edwards J, Dinh M, and Walton M. Reducing stress and burnout in junior doctors: the impact of debriefing sessions. Postgrad Med J. 2015 Apr;91(1074):182-7

6 Riley G. Understanding the stresses and strains of being a doctor. Med J Aust 2004; 181 (7): 350-353.

7 Williams E, Manwell L, Konrad T, and Linzer M. The relationship of organizational culture, stress, satisfaction, and burnout with physician-reported error and suboptimal patient care: Results from the MEMO study. Health Care Manage Rev. 2007 Jul-

Sep;32(3):203-12

8 Woolf S, Kuzel A, Dovey S, and Phillips R. A String of Mistakes: The Importance of Cascade Analysis in Describing, Counting, and Preventing Medical Errors. J Ann Fam Med. 2004; 2 (4) 317-326

9 Neale G, Woloshynowych M, and Vincent C. Exploring the causes of adverse events in NHS hospital practice. J R Soc Med. 2001 Jul; 94(7): 322-330.

10 James J. A New, Evidence-based Estimate of Patient Harms Associated with Hospital Care. J Patient Saf. 2013 Sep;9(3):122-8.

11 Kripalani S, LeFevre F, Phillips C, Williams M, Basaviah P, and Baker D. Deficits in Communication and Information Transfer Between Hospital-Based and Primary Care Physicians Implications for Patient Safety and Continuity of Care. JAMA. 2007;297(8):831-841.

12 Lawrence S, Spencer L, Sinnott M, and Eley R. It Takes Two to Tango: Improving Patient Referrals from the Emergency Department to Inpatient Clinicians. J Ochsner. 2015. 15(2);149-153

13 Houston J, Barker W, Clarke J, and Mew E. Sharing knowledge, saving time: an online toolbox to aid junior doctors. BMJ Qual Improv Report 2014;2

14 Tarrant M, Honeyman C, Aquilina A, and Young K. Improving the accessibility of trust guidelines for junior doctors at North Bristol NHS Trust. BMJ Qual Improv Report 2014;3

\section{Declaration of interests}

None declared 


\section{Acknowledgements}

To the foundation doctors of Tayside, thank you. Special mention to Nick Heath.

\section{Ethical approval}

This project was deemed an improvement study and not a study on human subjects. Local policy meant that ethical approval was not required. 